\title{
Reunión Anual de Investigación en su décima edición
}

L a enfermera, al ser el profesionista más próximo a la persona que se encuentra en un proceso de enfermedad, es aquella que en los últimos años ha tenido un impulso académico que se refleja y se proyecta a través de la presencia social y profesional en materia de salud, tanto en nuestro país como en el mundo. Es importante destacar que por ello los avances que son generados en materia asistencial, docencia y de gestión de los servicios de enfermería, se han difundido a través de la investigación mediante ciertos procesos y protocolos, los cuales han sido publicados en la Revista de Enfermería Neurológica que comenzó desde el año 2002. De inicio, únicamente eran divulgados los proyectos a investigar, sin embargo, a través de estos diez años han incursionado en este ámbito profesionistas enfermeras como la Lic. Nidia Coronado García, la Lic. Enf. Beatriz Trejo Casañas, la MEE. Araceli Sánchez Ramos, la MEE. María Guadalupe Nava Galán, la MCE. Araceli Jiménez Mendoza, que han contribuido y dado cimientos invaluables que en pleno siglo XXI dan credibilidad a la actual Revista de Enfermería Neurológica, que por iniciativa de la Enfermera Neuróloga Hortensia Loza Vidal se instauró y se lleva a cabo en la Unidad de Investigación e inició su primer ejemplar con el Vol. 1 Núm. 1 enero-diciembre, 2003. Hasta el momento, la revista ha tenido diferentes modificaciones en diseño, tanto en el interior como en el exterior. Sin embargo, lo más relevante es el avance en el contenido investigativo y creador de una revista científica que es difundida en bases de datos como CUIDEN, sustentado por el proceso editorial de Medigraphic, lo cual nos permite y proporciona visibilidad al accesar a la web; de igual forma, está siendo evaluada por Latindex, Redalyc para en un futuro cercano se visualice a través de plataformas como Scielo, LILACS, BIREME y Scopus, así como también para que se gestione lo propio para formar parte de la Biblioteca Virtual en Salud (BVS).

Este trabajo, como se mencionó con anterioridad, se lleva día con día en la Unidad de Investigación en Enfermaría (UIE), ubicada en el Edificio de Investigación Sociomédica, y representa desde hace ya varios años un espacio que promueve, fomenta, impulsa, desarrolla, promociona y difunde el avance científico del profesional de enfermería. Esto se ve respaldado por la presentación de conferencias en el marco de la investigación en salud en la Reunión de Investigación en Enfermería.

En un inicio, ésta se llevaba a cabo en un solo día, sin embargo, a consideración de la actual Subdirectora de Enfermería, la MRN. Rocio Valdez Labastida, y la producción en investigación que es recibida, se incrementaron a dos días. En el último lustro, se han tenido avances significativos en la proyección del evento coordinado hasta diciembre de 2013, por la MEE. María Guadalupe Nava Galán, Coordinadora de Investigación en Enfermería.

De manera simultánea, la Unidad de Investigación ha sido partícipe en la conformación de la Red de Investigación en Enfermería, siendo sede del VII Encuentro de la Red en octubre de 2013, como proyectos de investigación propiamente en red que están en proceso de conclusión y difusión de resultados, así como la instauración del proyecto Red de Editoras de Revistas Científicas en enfermería a partir de enero del presente año.

En esta ocasión, la $\mathbf{X}$ Reunión de Investigación en Enfermería se llevó a cabo los días 5 y 6 de junio, y de inicio contó con la presencia de dos conferencistas: el Mtro. Alejandro Machorro Nieves, quien como tercer vocal de la Asociación Mexicana de Editores de Revistas Biomédicas (AMERBAC), brindó la conferencia "Bioética en investigación y publicación”, y el Dr. Óscar Alberto Beltrán Salazar, Catedrático de la Universidad de Antioquia Colombia, que ofreció la ponencia "Modelo de reloj de arena: Esfuerzos humanizadores para el cuidado".

En este foro académico se presentaron 28 investigadores de diversas disciplinas, entre ellos doctores, doctorantes, maestras y licenciadas en enfermería, en educación, pedagogos, nutriólogos, psicólogos, químico farmacobiólogos, filósofas y especialistas en enfermería neurológica, quienes brindaron una diversidad de conocimiento de las diferentes disciplinas. Asimismo, se obtuvo un registro de 220 asistentes, tanto del personal del instituto como de diferentes instituciones 
de salud, tales como el Instituto Nacional de Cardiología, Instituto Nacional de Rehabilitación, Instituto Mexicano del Seguro Social, Unidad Médica de Alta Especialidad del Hospital de Pediatría, así como de universidades invitadas, entre ellas la Universidad Nacional Autónoma de México, la Escuela Nacional de Enfermería y Obstetricia (ENEO), quien en esta ocasión nos brindó y alojó en sus instalaciones, la Facultad de Estudios Superiores Zaragoza (FES Zaragoza), la Universidad Autónoma Metropolitana Unidad Xochimilco, la Benemérita Universidad de Puebla, la Universidad Tecnológica Nezahualcóyotl, la Universidad Veracruzana, la Universidad Autónoma del Estado de Morelos, Instituto de la Región Oriente, sede regional de la Universidad del Valle y Universidad Autónoma de Guanajuato.

Por la importancia del evento, en esta ocasión se otorgaron tres premios económicos proporcionados por la Fundación Armstrong, misma que desde su inicio avala y respalda este tipo de eventos con gran fuerza y distinción. Siendo los premiados:

$1^{\text {er }}$ lugar: Indicadores tempranos de dificultades del comportamiento en los primeros 2 años de vida por Est. Lic. Psic. Tania Ivonne Hernández Coloa, Dra. Dora Elizabeth
Granados Ramos y Ma. Magdalena Álvarez Ramírez de la Universidad Veracruzana.

$2^{\circ}$ lugar: Protocolo de manejo de paciente con corazón izquierdo hipoplásico por la Lic. Enf. María del Socorro Rodríguez Ramos y la Lic. Yolanda Velázquez Godoy del Instituto Nacional de Cardiología Ignacio Chávez.

$3^{\text {er }}$ lugar: Impacto en el nivel de eficiencia de los registros de atención de enfermería en cuidados críticos e intermedios posterior a un programa de Mejora por el Mtro. Noé Sánchez Cisneros del Instituto Nacional de Cardiología Ignacio Chávez.

Dichas investigaciones proyectan el hacer del cuidado a través de una mirada compartida y respaldada por las diferentes disciplinas e instituciones de educación así como de salud, que proporcionan y dan razón al propio cuidado, mismo que fundamenta el título de la X Reunión de Investigación en Enfermería.

\section{"Cuidado, una visión transdisciplinar"}

MCE. Angélica Guadalupe Dávalos-Alcázar 